\title{
Research and Analysis of Pitch Control Skills in Trumpet Playing
}

\author{
Wang Lei \\ Xi'an Conservatory of Music, Xi'an, Shaanxi, 710061
}

Key words: Trumpet performance; Pitch control; Research and analysis

\begin{abstract}
With the development of various industries in China, especially in the field of music, the communication between China and foreign countries has become increasingly frequent. In music field, the one that develops most rapidly is trumpet performance. As a brass-wind instrument, trumpet is an inseparable part in the performance of the entire team. Firstly, this paper analyzes the unique performance way of trumpet; then, according to changes of pitch, it studies techniques of pitch control.
\end{abstract}

\section{Introduction}

The sound of trumpet in performance is very resounding, and the color is very sharp. In the whole band, it can present a strong sense of splendor. The resounding sound in the band is played by trumpet, and it can also play out beautiful and fair-sounding melody according to requirements of temperament. In trumpet performance, in order to enhance the rhythm sense of trumpet, players usually use mute. Trumpet is very important in symphony orchestra and military band; meanwhile, it proposes higher requirements for trumpet. Trumpet is a wind instrument, and in the performance process, players need to be close to the trumpet so that air in the trumpet will vibrate. There are three pistons in the trumpet, whose function is to reduce the tone of trumpet effectively when air goes through the general length of the pipeline so that the tone diversity is enriched in the performance.

\section{Elements influencing pitch in trumpet performance}

Wind instrument developed slowly in China, and it sprung up and developed after the foundation of China, so the production of wind instrument was quite backward. The trumpet China produced after its foundation was very rough, and the quality was poorer than that in developed countries. If the quality of the trumpet was poor, it would have huge influence on the pitch and tone during performance. if the technological level of the trumpet was poor, the trumpet could not be used as professional instrument for performance; otherwise, it would reduce the integral performance standard of the band. Trumpet of poor quality could not conduct free stretching piston additional tube, so the pitch and tone could not be adjusted and modified effectively in performance.

Different performance environment could also cause influence on the pitch of the trumpet, and the temperature and humidity of the performance environment would also have interference on the pitch and tone. The vibration frequency of trumpet in performance would be influenced by the environment. Once the vibration frequency of trumpet is interfered by gravity environment, the pitch would also be changed of different degrees. In order to protect trumpet from environmental influence, players need to calibrate the pitch and tone of the trumpet to guarantee the temperature stays in a certain scope. The adjustment of pitch should also conform to environmental changes so 
that it would not be influenced by environment too much in the performance process; meanwhile, the trumpet's pitch would not be changed too much in performance. Trumpet is very sensitive to temperature and humidity. By the principle of thermal expansion, we know that the vibration frequency of trumpet is influenced by temperature so that when the trumpet is playing high pitch, the pitch would be deviated. If we want to avoid such phenomenon, we can calibrate the temperature of the trumpet, so when the trumpet's temperature reaches a certain scope, after some calibration of the pitch, the trumpet will not have pitch deviation problems in the performance.

The performers' playing standard would also influence the play of trumpet in the performance. If the players' performing standard is high, when the trumpet's pitch is deviated because of environmental influences, players can control the pitch according to their previous performing experiences. The trumpet also has strict requirements on players. Once the player's mouth shape is deviated a little during performance, the breath would be different obviously, which would lead to errors of pitch. If it is players with rich experiences of trumpet playing, when the pitch is not right, they will calibrate the trumpet rapidly to guarantee the pitch is in a certain scope. The closing degree of the player's mouth would also influence the pitch of the trumpet to some extent. Different music also has different requirements on the closing degree of the player's mouth. If trumpet player's closing degree of mouth is different, the music would also be different in pitch and music score; meanwhile, the performance standard of the whole band would also be different. The player's breath control would also influence the pitch of the trumpet. When playing trumpet, the breath must be grasped stably. During performance, requirements on players are also very high, and players need to use stable breath to express music during the whole course. If the breath fluctuates greatly, noises would appear before the trumpet makes any sound. The noise would influence the integral musicality of the trumpet severely. The trumpet player's auditory sensitivity would also influence the pitch of the trumpet. When the trumpet is influenced by external environment and deviations appear, if players can distinguish the specific position of the pitch rapidly, and calibrate the pitch in time according to previous performance experiences, the performance effects of the whole band would not be influenced. Therefore, trumpet player's auditory training is very important in daily training. In player's normal exercise, tests in pitch should be enhanced to improve player's sensitivity to pitch.

Trumpet is a copper pipe instrument, and 12 equal temperament is adopted. Most of other instruments adopt pure tone temperament or 5 pitch generating temperament, and 12 equal temperament is different from other two rhythms. At present, the manufacturing technique of trumpet in China is not complete yet, and the system of manufacturing streamline is not perfect. Through the manufacturing standard of trumpet has improved obviously, compared with manufacturing standard of foreign instrument, it is still difficult to control the pitch. Trumpet has its own unique tuning system, so it is more difficult for players to control the pitch of the trumpet compared with other instruments. Especially on trumpet, it is very difficult to control the pitch. If players with ordinary playing standard meet trumpet of bad quality, it is extremely hard for them to control the pitch. If players cannot control the trumpet with bad quality, they will use substitute keys to replace the pitch, which would improve the performance standard of the whole band to some extent.

\section{Control skills of pitch in trumpet playing}

During performance, various accidents would happen, which requires trumpet players to accumulate many experiences so that they can adjust in time when anything happens in future performance. If accidents happen in the performance, it would be difficult for players to control the 
pitch within a certain scope, which would lead to the failure of the whole performance. In daily practice, players' listening practice must be strengthened so that their perception of pitch will be enhanced; furthermore, in the performance process, if accidents happen, players can locate the accident and deviation instantly. In daily listening practice, training of pitch is very important. In all of the current listening practice courses, the most effective training method is to have the players learn solfeggio and ear training course. Solfeggio and ear training is the most practical course, and it can improve players' listening effectively. When players' listening is improved to a certain standard, they can calibrate the deviated pitch in time during the performance. If players have not received professional listening training, even if they can find pitch problems in the performance, they cannot adjust the deviated pitch within effective time so that the performance effects of the whole band would be severely influenced. In daily practice, listening practice can be strengthened for players so that their perception of pitch can be improved to a new level. In all the current courses of training to players, listening practice is very effective. After the training, players can improve their listening sensitivity to the pitch of the trumpet effectively and find problems of pitch during performance process.

To practice an instrument, one has to train basic content. Only by grasping basic training content can later study and development be promoted. In the training of basic skills, the quality of the class must be guaranteed. During the study, one has to stick to the end and study hard. Dropping out halfway should be prohibited. As for study of trumpet, the control of pitch is very important. During daily study, basic training of pitch must be paid attention to. During the practice, changes of mouth shape and adjustment of breath should be noticed. In combination with one's own characteristics, accuracy of the pitch should be guaranteed. At the same time, frequency of prolonged sound should be improved during training. During trumpet playing process, the control of prolonged sound is very difficult, and special exercise is needed in this aspect. In order to control the performance of prolonged sound better, players need to find a method suitable for themselves and find scientific breathing method, thereby guaranteeing that they have sufficient breath for adjustment. Thoracicabdominal respiration is very suitable. Breath is usually speeded up in trumpet playing, so the pitch would be risen; meanwhile, trumpet player's mouth is tightened, which leads to the increase of pitch. If player loosens his mouth during performance, the pitch would be decreased substantially. These experiences should be summed up during daily practice and be applied in future performance, thereby controlling the stability of pitch in performance effectively. In the practice, the training of prolonged sound should be put at last, since in this way, previous training experiences can be concluded, and previous training can be applied in later training, which can improve player's training efficiency effectively.

Different players have their unique playing methods and techniques, and their habitual playing methods and habits might also be different. During the performance, different players would also have different mouth shapes and breath. However, besides techniques of mouth shape and breath, players should also adjust their own playing condition according to their own performance characteristics. During the performance, the success of the performance lies in the accuracy of pitch. If players can control the pitch within a certain scope, they can crate a high-standard performance atmosphere and effects. Besides strengthening pitch control in daily practice, players should also be good at using piston additional tube, which can control changes of pitch effectively to some extent. Many interference factors would appear in the performance of trumpets. As trumpet players, they would also have their own unique playing methods and habits, and different trumpet players have different mouth shapes. Therefore, players must grasp breath skills and mouth shape changes and take advantage of piston additional tube flexibly in the performance. In this way, they can calibrate 
the pitch effectively and guarantee the optimal performance condition in the performance.

\section{Conclusion}

Trumpet playing develops the most rapidly in the field of music. As a brass-wind instrument, trumpet can present a strong sense of splendor in the whole band. Trumpet is a wind instrument. During the performance, players need to be close to the trumpet, and the air in the trumpet would vibrate. In order to better control the performance of medium-length notes, players need to find methods that are suitable to them. If the manufactured trumpet is of bad quality, the trumpet would not be suitable for professional performance. In order to prevent the trumpet from being influenced by environment, players need to calibrate the pitch and tune of the trumpet before playing.

\section{Bibliography:}

[1] Chen Jiebin. Research and Analysis of Pitch Control Skills during Trumpet Playing[J]. Northern Music,2014(15).

[2] Fu Shaowei. Control Skills of Pitch during Trumpet Playing [J]. Music Space Time,2015(06).

[3] Wang Lei. On Breath in Trumpet Playing [J]. Shaanxi Education (Higher Education Edition),2011,(9).

[4] He Wenjing. On Problems and Countermeasures of Pitch in Trumpet Playing [J]. Popular Art (Theory),2009,(7). 